\title{
An improved method for the preparations of nanostructured lipid carriers containing heat-sensitive bioactives.
}

\begin{abstract}
Heat-sensitive bioactive compounds such as $\beta$-carotene and tocols, are widely used in the pharmaceutical and cosmetic fields. Their chemical stability in delivery systems is one of the major concerns in the production of nanostructured lipid carriers (NLCs). A previously established high-temperature high-pressure homogenisation technique involved in the preparation of NLCs can cause degradation of heat-sensitive compounds. Therefore, a novel preparation process needs to be developed to minimise the degradation of heat-sensitive active compounds during the preparation of NLCs. In this work, modified methods A and B were designed to minimise the degradation of $\beta$-carotene and tocols during the production of NLCs. These methods improved the chemical stability of heat-sensitive bioactive compounds ( $\beta$-carotene and tocols) significantly compared to the previously established method. The physical stability of the formulation was maintained throughout study duration.
\end{abstract}

Keyword: Improved method; Preparations; Nanostructured lipid carriers; Heat-sensitive bioactives. 